\title{
Mechanismen der kardiovaskulären Komorbidität bei Patienten mit obstruktivem Schlafapnoesyndrom
}

\author{
Mechanisms of Cardiovascular Co-Morbidity in Patients with Obstructive Sleep \\ Apnoea Syndrome
}

Autoren

Institute
M. Meier ${ }^{1}$, S. Andreas ${ }^{1,2}$

${ }^{1}$ Lungenfachklinik Immenhausen/Krs. Kassel

${ }^{2}$ Abtl. Kardiologie und Pneumologie, Universitätsmedizin Göttingen

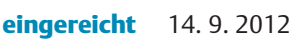
akzeptiert nach Revision 19. 9.2012

Bibliografie

Dol http://dx.doi.org/

10.1055/s-0032-1325788

Pneumologie 2012; 66: 650-657

(c) Georg Thieme Verlag KG Stuttgart · New York ISSN 0934-8387

Korrespondenzadresse Prof. Dr. med. S. Andreas Lungenfachklinik Immenhausen Pneumologische Lehrklinik Universität Göttingen Robert-Koch-Straße 3 34376 Immenhausen sandreas@lungenfachklinikimmenhausen.de

\section{Zusammenfassung \\ $\nabla$}

Die bei obstruktiver Schlafapnoe repetitiv auftretende intermittierende Hypoxie, zentralnervöse Weckreaktion und intrathorakale Druckschwankung sind als wesentliche pathophysiologische Mechanismen für die kardiovaskuläre Komorbidität verantwortlich. Diese Phänomene führen zu vermehrtem oxidativen Stress, systemischer Inflammation, Störungen der Vasomotorik, Steigerung sympathischer Aktivität und Arteriosklerose der Blutgefäße. Die CPAP-Therapie senkt den arteriellen Blutdruck und hat einen positiven Effekt auf wesentliche Einzelaspekte der kardiovaskulären Komorbidität. Vor der Veröffentlichung bereits laufender randomisierter Studien werden wir im klinischen Alltag im Einzelfall entscheiden müssen, ob allein die kardiovaskuläre Situation eine CPAP-Therapie rechtfertigt. Dies wird insbesondere dann der Fall sein, wenn eine schwere OSA vorliegt, eine Tagesmüdigkeit besteht, eine gute Compliance gegeben ist und die Patienten jünger sind. Auf jeden Fall sinnvoll sind Maßnahmen, die das kardiovaskuläre Risiko senken und dabei gleichzeitig einen positiven Einfluss auf die OSA haben, d. h. Gewichtsreduktion, körperliches Training und Tabakentwöhnung.

\section{Einleitung}

\section{$\nabla$}

Die obstruktive Schlafapnoe (OSA) ist durch wiederholte komplette oder inkomplette Einengungen der oberen Atemwege während des Schlafes charakterisiert, die zu vermehrten Atemanstrengungen mit verstärkten intrathorakalen Druckschwankungen, zu alveolärer Hypoventilation mit konsekutiver intermittierender Hypoxie und zu Weckreaktionen mit daraus resultierender Tagesschläfrigkeit führen. Hauptursache der OSA ist die Adipositas, die über eine Vermehrung insbesondere der lateralen Fettpolster den PharynxQuerschnitt reduziert [1]. Eine 10\%ige Gewichts-

\section{Abstract \\ $\nabla$}

The repetitive intermittent hypoxic episodes, central nervous arousal and intrathoracic pressure fluctuations that occur together with obstructive sleep apnoea are the primary pathophysiological mechanisms responsible for the cardiovascular co-morbidity. These phenomena lead to increased oxidative stress, systemic inflammation, disrupted vasomotor function, increased sympathetic activity and the development of arteriosclerosis in blood vessels. CPAP therapy lowers arterial blood pressure and has a positive effect on relevant specific aspects of cardiovascular comorbidity. Until the results of current randomised studies have been published, it has to be decided on an individual basis whether the cardiovascular situation alone justifies CPAP therapy. This will usually be the case in severe OSA with daytime sleepiness, in young patients and patients with good compliance. Interventions that reduce cardiovascular risk and also have a positive effect on OSA, i.e., weight reduction, physical exercise and smoking cessation are always appropriate.

zunahme erhöht das OSA-Risiko um den Faktor 6 [2]. Angesichts der epidemisch auftretenden Adipositas wird die Prävalenz der OSA und des metabolischen Syndroms weiter steigen. Trotzdem soll erwähnt werden, dass auch knöchern-anatomische und hereditäre Faktoren eine Rolle in der Pathogenese der OSA spielen. 


\section{Epidemiologie}

$\nabla$

\section{OSA}

In der US-amerikanischen Studie zur Prävalenz der OSA, der Wisconsin Sleep Cohort Study, in der 602 Personen im Alter von 30 bis 60 Jahren polysomnografisch untersucht wurden, zeigten $5 \%$ aller Frauen und 15\% aller Männer über 10 Apnoen oder Hypopnoen pro Stunde Schlaf [3]. Bei $4 \%$ bzw. $2 \%$ traten mehr als 5 Apnoen oder Hypopnoen pro Stunde Schlaf sowie eine erhöhte Tagesschläfrigkeit auf. Eine aktuellere spanische Untersuchung kommt zu vergleichbaren Zahlen [4]. Ohne gleichzeitiges Vorliegen einer Tagesschläfrigkeit hatten 26\% der Männer und 28\% der Frauen im Alter von 30 - 70 Jahren einen AHI $\geq 5$ und 14\% bzw. 7\% einen $\mathrm{AHI} \geq 15$ [4]. Wie zu erkennen, sind Männer häufiger als Frauen betroffen. Nach der Menopause gleicht sich die Prävalenz der Geschlechter jedoch an.

\section{Kardiovaskuläre Erkrankungen}

Kardiovaskuläre (KV) Erkrankungen stellen in der westlichen Welt eine erhebliche gesundheitsökonomische Herausforderung dar. Obwohl sich die standardisierte Sterblichkeit für KV-Erkrankungen von 1998 bis 2008 in Deutschland reduzierte, ist sie sowohl bei Frauen mit 46,6\% als auch bei Männern mit 37,3\% die Haupttodesursache (Statistisches Bundesamt, Todesursachenstatistik). Besonders ältere Menschen leiden an KV-Erkrankungen. Die wesentlichen KV-Erkrankungen sind die ischämische Herzerkrankung, die Herzinsuffizienz und zerebrovaskuläre Erkrankungen bzw. der Schlaganfall.

\section{Ischämische Herzerkrankung}

Obwohl die Sterblichkeit an der ischämischen Herzkrankheit in den letzten 10 Jahren leicht abgenommen hat, bleibt die ischämische Herzkrankheit die Haupttodesursache mit einer Inzidenz von etwa 400 Myokardinfarkten/100.000 Einwohnern/Jahr in der Altersgruppe der 36-64-Jährigen. Der Anteil der an ischämischer Herzkrankheit Verstorbenen an der Gesamtheit der durch kardiovaskuläre Erkrankungen Verstorbenen beträgt bei Frauen $33 \%$ und bei Männern 45\%, wohingegen der Anteil zerebrovaskulärer Erkrankungen bei Männern 16\% und bei Frauen 19\% ausmacht.

\section{Herzinsuffizienz}

Die Prävalenz der Herzinsuffizienz beträgt etwa $2 \%$, steigt jedoch bei Personen $>70$ Jahre auf $>10 \%$ an. Entsprechend ist das mittlere Alter von Patienten mit Herzinsuffizienz 75 Jahre. Dabei weist etwa die Hälfte der Patienten mit den klinischen Zeichen der Herzinsuffizienz eine unauffällige linksventrikuläre Auswurfsfraktion auf [5]. Die Erkrankung dieser Patienten wird als Herzinsuffizienz mit erhaltener systolischer Funktion definiert, wobei im Allgemeinen eine gestörte diastolische linksventrikuläre Funktion als zugrunde liegende Ursache angesehen wird. Ursachen sind arterielle Hypertonie, Diabetes mellitus und Vorhofflimmer [5]. Die Prognose der stationär behandelten diastolischen Herzinsuffizienz ist vergleichbar mit der Prognose der systolischen Herzinsuffizienz. Es fehlen therapeutische Strategien, die über die Kontrolle von Risikofaktoren wie der arteriellen Hypertonie hinausgehen.

\section{Metabolisches Syndrom als kardiovaskulärer} Risikofaktor

Das metabolische Syndrom ist eng mit Adipositas und ihren Folgen assoziiert. Entsprechend der Konsensuskonferenz von 2009 wird das metabolische Syndrom über mindestens 3 der folgenden Merkmale definiert: Taillenumfang bei Frauen $\geq 88 \mathrm{~cm}$, Hüftumfang bei Männern $\geq 102 \mathrm{~cm}$; erhöhter arterieller Blutdruck (systolisch $\geq 130$ oder diastolisch $\geq 85 \mathrm{mmHg}$ ) oder antihypertensive Therapie; erhöhte Nüchternblutglukose $(\geq 5,6 \mathrm{mmol} / \mathrm{l})$ oder antidiabetische Therapie; erhöhte Triglyceride $(\geq 1,7 \mathrm{mmol} / \mathrm{l})$ oder lipidsenkende Therapie; erniedrigtes HDL-Cholesterin ( $<1,3 \mathrm{mmol} / \mathrm{l}$ bei Männern und $<1 \mathrm{mmol} / \mathrm{l}$ bei Frauen) oder lipidsenkende Therapie. Das weiße Fettgewebe ist ein wichtiges endokrin wirksames Organ und spielt eine Schlüsselrolle in der Entwicklung des metabolischen Syndroms. Bei Adipositas ändert und steigert sich die inkretorische Aktivität des Fettgewebes. Als mögliche Ursache wird eine relative Hypoxie von Fettzellnestern, die sich mit zunehmender Größe von den versorgenden Gefäßen entfernen, angenommen. Die negativen kardiovaskulären Effekte werden überwiegend von intraabdominellem bzw. visceralem Fettgewebe und nicht von subkutanem Fettgewebe bedingt.

\section{OSA ist bei KV-Erkrankungen häufig}

Eine OSA findet sich häufig bei der ischämischen Herzkrankheit, Herzinsuffizienz und Schlaganfall. Mehrere Studien an Patienten mit ischämischer Herzkrankheit und gleichzeitig vorliegendem leichten Übergewicht ergaben eine Häufigkeit der OSA von 30\%$50 \%$ [6]. Mit großer Häufigkeit wird die OSA ebenfalls bei Patienten mit arterieller Hypertonie, Schlaganfall und Herzinsuffizienz gefunden [7] ( Tab. 1). OSA und KV-Erkrankung teilen die gleichen Risikofaktoren wie Adipositas, männliches Geschlecht und zunehmendes Alter.

Tab. 1 Häufigkeit der OSA bei verschiedenen KV-Erkrankungen (nach [6, 7]).

\begin{tabular}{|l|l|}
\hline koronare Herzerkrankung & $30-60 \%$ \\
\hline Herzinsuffizienz & $10-50 \%$ \\
\hline arterieller Hypertonus & $30-80 \%$ \\
\hline Schlaganfall & $40-90 \%$ \\
\hline
\end{tabular}

\section{Ischämische Herzkrankheit}

Die Prävalenz der OSA beträgt bei Patienten mit stabiler ischämischer Herzkrankheit je nach Alter und Begleiterkrankungen bis zu 60\% ( Tab.1). Hung et al. haben erstmalig Patienten nach Myokardinfarkt untersucht und fanden einen mittleren AHI von $13 / \mathrm{h}$, wohingegen der AHI bei männlichen Kontrollen ohne Anhalt für ischämische Herzkrankheit lediglich $4 / \mathrm{h}$ betrug [8]. Kürzlich konnte die Arbeitsgruppe aus Regensburg zeigen, dass im Zuge der regelmäßig zu beobachtenden Verbesserung der linksventrikulären Funktion nach Myokardinfarkt sich auch die OSA verbessert [9].

\section{Herzinsuffizienz}

Nach einer aktuellen multizentrischen Untersuchung der $\mathrm{Ar}$ beitsgruppe Kreislauf und Schlaf der Deutschen Gesellschaft für Schlafforschung und Schlafmedizin wurden bei Patienten mit Herzinsuffizienz zu über $60 \%$ schlafbezogene Atmungsstörungen (SBAS) gefunden [10]. Die SBAS manifestieren sich zu jeweils etwa gleichen Anteilen in zentralen Atmungsstörungen in Gestalt der Cheyne-Stokes-Atmung (CSA) und der OSA [10,11]. Bei 
übergewichtigen Kollektiven mit Herzinsuffizienz wird die OSA und bei kachektischen Kollektiven eher die CSA im Vordergrund stehen.

\section{Akute Effekte obstruktiver Apnoen \\ $\nabla$}

Blutdruck Die Apnoe führt über zwei unabhängige Mechanismen zu einem Anstieg des arteriellen Blutdruckes. Zum einen bewirkt die progrediente hyperkapnische Hypoxie über Chemorezeptoren eine sympathische Aktivierung. Hierfür spricht, dass der Blutdruckanstieg während Apnoen durch Gabe von Sauerstoff vermindert werden kann [12,13]. Neben diesen Änderungen der Blutgase führt auch die zunehmende mechanische Atemarbeit zu einer zentral-nervösen Arousalreaktion, die einerseits die Apnoe beendet und andererseits zu einem Blutdruckanstieg führt. Z.B. führen Arousalreaktionen, die durch eine Vibration des Kopfkissens ausgelöst wurden, zu einem Anstieg des Blutdruckes [14]. Insgesamt ist es daher verständlich, dass Patienten mit OSA einen fehlenden nächtlichen Abfall des arteriellen Blutdruckes zeigen, während bei gesunden Personen in der Nacht der arterielle Blutdrucke um 10-20\% abfällt.

Negativer intrathorakaler Druck Die pharyngeale Obstruktion bei weiterhin erhaltener Tätigkeit der Atemmuskulatur verursacht eine intermittierende Negativierung des intrathorakalen Druckes, die mit Dauer der Apnoe zunimmt und häufig Werte von - 50 mm Quecksilbersäule erreicht. Der negative intrathorakale Druck (Müller-Manouver) führt zu einer kurzfristig vermehrten Füllung des rechten Atriums und des rechten Ventrikels. Weiter nimmt aufgrund der Erhöhung der links-ventrikulären Nachlast (durch den negativen intrathorakalen Druck) das linksventrikuläre Schlagvolumen ab und die diastolische linksventrikuläre Funktion wird gestört [15]. Entsprechend fallen das LVenddiastolische Volumen und die EF insbesondere bei bereits geschädigtem linken Ventrikel ab [16].

Herzfrequenz Während der obstruktiven Apnoe kommt es wahrscheinlich durch Exzitation pharyngealer Mechanorezeptoren und bei steigendem Blutdruck über den Baroreflex zu einem Abfall der Herzfrequenz [17]. Dieser wird mit zunehmender Dauer der Apnoe durch die auftretende Sauerstoffentsättigung und Aktivierung des sympathischen Systems jedoch z.T. aufgehoben [18]. Trotzdem werden Bradyarrhythmien bis hin zum AV-Block III ${ }^{\circ}$ beobachtet.

Myokardiale Ischämien Während obstruktiver Apnoen fällt die Herzfrequenz sowie das linksventrikuläre Schlagvolumen und somit das Herzzeitvolumen. Dabei steigt die Nachlast und die Sauerstoffsättigung fällt. Am Ende der Apnoe und der dann folgenden Arousalreaktion kommt es zu einem weiteren Anstieg der links-ventrikulären Nachlast und der Herzfrequenz. Hiermit verbunden steigt der myokardiale Energieverbrauch gerade dann an, wenn die arterielle Sauerstoffsättigung niedrig ist. Gesunde Herzkranzgefäße können durch Vasodilatation die myokardiale Perfusion und damit das Sauerstoffangebot erheblich vergrößern. Daher tritt bei herzgesunden Patienten keine Myokardischämie auf $[19,20]$. Bei bestehender koronarer Herzerkrankung ist die OSA jedoch die Hauptursache für das Auftreten der nächtlichen Angina pectoris [21].

Zerebrale Gefäße Der Widerstand der intrazerebralen Gefäße wird wesentlich durch den Kohlendioxid-Partialdruck beeinflusst. Eine Hyperkapnie, die am Ende der Apnoe auftritt, führt zu einer Abnahme des Widerstandes und einer Zunahme des zerebralen Blutflusses, zumal es am Ende der Apnoe auch zu einer

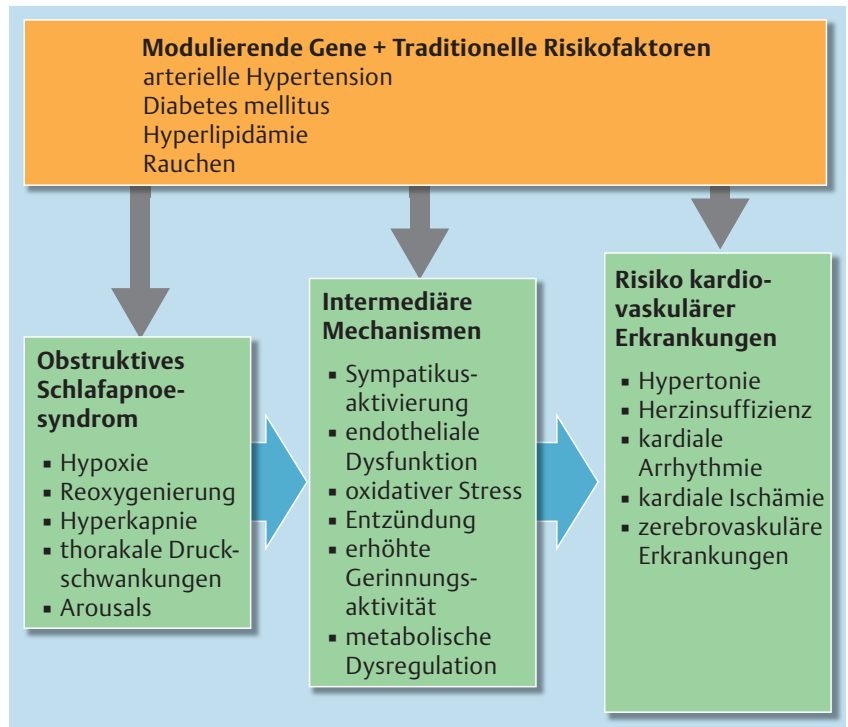

Abb.1 Zusammenhang zwischen obstruktiver Schlafapnoe und kardiovaskulärem Risiko (modifiziert nach [6] und [84]).

Zunahme des arteriellen Blutdruckes kommt. Mittels Spektroskopie konnte nachgewiesen werden, dass die zerebrale Oxygenierung infolge der Hypoxie bei OSA abfällt [22].

Pulmonaler Druck Die während der Apnoen auftretende Hypoxie führt über den Euler-Liljestrand-Mechanismus zur Vasokonstriktion [23]. Wie oben beschrieben, bewirkt der negative intrathorakale Druck eine Erhöhung des rechtsventrikulären Schlagvolumens und wohl auch des transmuralen linksventrikulär-enddiastolischen Druckes. Da bei zentralen Apnoen häufig kein Anstieg des PA-Druckes festzustellen ist und die Gabe von Sauerstoff den Anstieg des PA-Druckes nur zum Teil verhindert, ist die Hypoxie nicht der alleinige Mechanismus, der zu einem Druckanstieg im kleinen Kreislauf führt.

\section{Intermediäre Mechanismen}

$\nabla$

Im vergangenen Jahrzehnt hat die Forschung zahlreiche negative Auswirkungen der OSA auf das kardiovaskuläre System aufgedeckt. Die pathophysiologische Interaktion zwischen OSA und KV-Erkrankungen ist komplex und umfasst neurale, humorale, mechanische, hämodynamische und zirkadiane Komponenten ( $\bullet$ Abb. 1). Oxidativer Stress und endotheliale Dysfunktion sind die Hauptmechanismen, die bei der OSA zu KV-Erkrankungen führen. Ein weiterer wichtiger Effekt ist eine über den Tag persistierende Zunahme der Sympathikusaktivität. Gut kontrollierte Studien haben Auswirkungen der OSA auf unterschiedliche Aspekte der frühen vaskulären Dysfunktion aufgezeigt. Hierzu gehören die Gefäßreaktionen auf unterschiedliche Stimuli, arterielle Steifigkeit, Pulswellengeschwindigkeit, Intima-Media-Dicke der Carotis, Arterienplaques in den Carotiden und Veränderungen der Koronararterien. Diese Veränderungen hängen letztendlich mit klinisch manifester KV-Erkrankung in verschiedenen Gefäßbetten zusammen. Die wesentlichen Mechanismen werden nachfolgend besprochen.

Oxidativer Stress Die im Rahmen der OSA auftretenden intermittierenden Hypoxien führen entsprechend dem Modell von Ischämie und Reperfusion zu einer vermehrten Produktion freier Sauerstoffradikale [24], die zu einer Inflammation und reduzier- 
ter Verfügbarkeit endothelialen NOs führt [25]. Diese Veränderungen sind unter CPAP-Therapie vollständig reversibel.

Inflammation Aus Untersuchungen an Zellkulturen ergibt sich, dass durch intermittierende Hypoxie über den hypoxieinduzierbaren Faktor (HIF)-1 entzündliche Kaskaden angestoßen werden, die zu vermehrter Produktion von Tumornekrosefaktor (TNF), Interleukin (IL)-8 und interzellulärem Adhäsionsmolekül 1 (ICAM1) führen können [26,27]. Bezüglich proinflammatorischer Zytokine, wie des C-reaktiven Proteins (CRP) und des (IL)-6 konnten erhöhte Spiegel bei Patienten mit obstruktivem Schlafapnoesyndrom ermittelt werden, die sich unter nCPAP-Therapie normalisierten [28].

Vasoaktive Substanzen In Tierversuchen konnte gezeigt werden, dass intermittierende Hypoxie mit erhöhtem Spiegel von Endothelin-1, einem vasokonstriktorischen Neuropeptid, erhöhter Expression von Endothelin-1-Rezeptoren und erhöhter vasokonstriktorischer Empfindlichkeit gegenüber Endothelin-1 assoziiert ist [29]. Bei Patienten mit obstruktiver Schlafapnoe jedoch konnten nicht durchgehend erhöhte Endothelin-1-Spiegel gemessen werden [30,31]. Auch gibt es Hinweise auf erhöhte Plasmaspiegel anderer vasoaktiver Substanzen wie Angiotensin II, Aldosteron und Renin.

Hyperkoagulabilität Zusätzlich trägt die Hyperkoagulabilität über eine verstärkte Gerinnungsneigung zur Arteriosklerose bei. Bei OSA-Patienten wurden erhöhte Werte von Hämatokrit, Fibrinogen, Blutviskosität und Plasminogen-Aktivator-Inhibitor-1 (PAI-1) bestimmt $[32,33]$. Unter CPAP kommt es zu einer Normalisierung dieser Veränderungen [34].

Autonomes Nervensystem Im Tierversuch konnte bei Exposition gegenüber intermittierender Hypoxie eine erhöhte Produktion von Kateocholaminen bei ansteigenden Blutdruckwerten gemessen werden. Bei Menschen konnte eine erhöhte sympathische Aktivität auch am Tage mittels der Mikroneurografie gezeigt werden $[35,36]$. Diese Sympathoexitation wird auch durch die erhöhte Tagesschläfrigkeit vermittelt [37], ist durch CPAP reversibel und hat multiple negative Folgen z.B. auf die „Uhren-Gene“ bzw. „innere Uhr“ [38], Muskeldysfunktion und vor allem auf das KV-System [39].

Endotheliale Dysfunktion und Arteriosklerose Die endotheliale Dysfunktion wird über eine gestörte Balance zwischen lokal wirksamen vasokonstriktorischen und vasodilatatorischen Substanzen definiert. Die vaskulären Veränderungen bei der OSA werden durch arterielle Hypertonie, durch Hypoxie vermittelten oxidativen Stress, systemische Inflammation, vermehrter Freisetzung vasoaktiver Substanzen, erhöhter sympathischer Aktivität sowie erhöhter transmularer Wandspannung intrathorakaler Gefäße und daraus resultierendem Wandstress bedingt. Z.B. konnten bei ansonsten gesunden OSA-Patienten eine verminderte endothel-vermittelte Vasodilatation nach intraarterieller Infusion von Acetylcholin sowie verminderte NO-Spiegel $[25,40]$ und eine reduzierte Anzahl endothelialer Progenitorzellen im Blut nachgewiesen werden. Auch repetitive Arousals beeinträchtigen die endotheliale Funktion [41]. Konsekutive Befunde bei ansonsten gesunden Patienten mit OSA sind eine gestörte Mikrozirkulation [42] und eine verdickte Intima der A. carotis [43].

Metabolisches Syndrom Der Schlaf beeinflusst die Adipozytenaktivität und das metabolische Syndrom. Diese Phänomene können durch einen proinflammatorischen Zustand aufgrund von Stress und neurohumoraler Aktivierung erklärt werden. In einem breiteren Zusammenhang erklärt die „selfish brain theory“", dass chronischer Stress die Mechanismen, mit denen das Gehirn seine eigene Energieversorgung sichert, beeinflusst [44]. Dadurch wird die Energieaufnahme der meisten Personen andauernd gesteigert [44].

Die Mechanismen, über die die OSA den Metabolismus negativ beeinflusst, sind komplex und umfassen sympathische und neurohumorale Aktivierung, die Glukosehomöostase, Inflammation und oxydativen Stress. Marker des metabolischen Syndroms wie Leptin sind erhöht und korrelieren mit dem Schweregrad der OSA. Große Querschnittsuntersuchungen schätzen das Risiko für das metabolische Syndrom bei Patienten mit OSA gegenüber Kontrollen als mindestens verdoppelt ein [45].

Bei Patienten mit OSA, jedoch ohne Diabetes mellitus Typ 2 und damit überwiegend ohne Adipositas, konnte CPAP die Glukoseeinstellung verbessern und die Insulinsensitivität steigern. Bei dem typischen übergewichtigen Patienten mit OSA beeinflusst CPAP den metabolischen Status jedoch nicht wesentlich. Kürzlich zeigte eine randomisierte, kontrollierte Crossover-Studie an 86 Patienten aus Indien überzeugend, dass eine dreimonatige CPAP Therapie signifikante positive Effekte auf den Blutdruck, Hba1c, Cholesterin, Triglyceride und insgesamt das metabolische Syndrom hatte [46]. Diese Ergebnisse erinnern an die Situation bei KV-Erkrankungen, bei denen die Effekte von CPAP am deutlichsten bei jungen, ansonsten gesunden männlichen Patienten mit OSA nachgewiesen wurden.

\section{Zentrale Apnoen unterscheiden sich von obstruktiven Apnoen \\ $\nabla$}

Insbesondere bei der Herzinsuffizienz treten repetitive zentrale Apnoen auf, die meist als Cheyne-Stokes-Atmung (CSA) beschrieben werden. Als Ursache der CSA gilt eine Oszillation des rückgekoppelten Regelkreises der Atmung. Die initiale Hypothese, dass die CSA analog der OSA ausgeprägte negative Effekte auf das KVSystem hat [47], konnte bisher nicht bestätigt werden. Auch ist für die CSA der von der OSA bekannte enge Zusammenhang mit der arteriellen Hypertonie, der Aktivierung des autonomen Nervensystems [48] und der Tagesmüdigkeit nicht belegt. Dies ist nicht verwunderlich, da es bei zentralen Apnoen nicht zu intrathorakalen Druckschwankungen kommt. Auf keinen Fall können wir die bei der OSA gewonnenen Einsichten ohne Weiteres auf die CSA übertragen.

\section{OSA verursacht arteriellen Hypertonus \\ $\nabla$}

Im Tierversuch besteht ein ausgeprägter Zusammenhang zwischen nächtlichen obstruktiven Apnoen und am Tage persistierendem arteriellen Hypertonus [49]. Ergebnisse epidemiologischer Untersuchungen zeigen, dass die obstruktive Schlafapnoe ein unabhängiger Risikofaktor für das Vorliegen einer arteriellen Hypertonie ist. Zwei Metaanalysen, die Patienten mit normalen und erhöhten Blutdruckwerten einschlossen, konnten eine Blutdrucksenkung von $2-3 \mathrm{mmHg}$ unter CPAP-Therapie zeigen [50, 51]. Insbesondere jüngere Patienten mit höherem $\mathrm{AHI}$ und guter Compliance zeigen einen deutlichen Effekt der CPAP-Therapie auf den arteriellen Blutdruck. Hingegen ist der Effekt bei OSA Patienten ohne Tagesschläfrigkeit sehr gering [52]. 


\section{CPAP: Positive Effekte auf KV-Erkrankungen} $\nabla$

Arterielle Hypertension ist ein Risikofaktor für die koronare Herzkrankheit und für die Herzinsuffizienz, v.a. die Herzinsuffizienz mit erhaltener Ejektionsfraktion, und ist auch ein starker Risikofaktor für Schlaganfall. Andererseits zeigten groß angelegte, epidemiologische Querschnitt- und Längsschnittstudien einen Zusammenhang zwischen OSA und KV-Morbidität und Mortalität, die von Confoundern wie Adipositas oder arterieller Hypertension unabhängig war. Die Wahrscheinlichkeit, an kardiovaskulären Ursachen zu versterben, ist doppelt so hoch bei Patienten mit mäßiger bis schwerer OSA, unabhängig von anderen Risikofaktoren wie Adipositas und arterieller Hypertension. Dieser Zusammenhang wurde für zahlreiche wesentliche Endpunkte nachgewiesen, wie Tod durch KV-Ereignis, akuter Myokardinfarkt, Schlaganfall, Auftreten von Herzinsuffizienz, stationäre Aufnahme wegen KV-Erkrankung, linksventrikuläre diastolische Dysfunktion, komplexe ventrikuläre Arrhythmien und plötzlicher Herztod.

In einer Reihe von großen, nicht randomisierten, retrospektiven epidemiologischen Untersuchungen erbrachte die CPAP-Therapie eine Reduktion der KV-Mortalität um 50-70\% [6,53-55]. Dabei scheinen insbesondere Männer mit einem Alter unter 70 Jahren und einem Apnoe-Hypopnoe-Index von > 20-30/Std. zu profitieren $[56,57]$. Die positiven Effekte der CPAP-Therapie werden im Folgenden für einzelne KV-Erkrankungen dargelegt (siehe auch $\bullet$ Tab.2).

Linksherzinsuffizienz Die diskutierten Folgen der OSA, insbesondere der arterielle Hypertonus, sind für den bereits geschädigten linken Ventrikel bei der Herzinsuffizienz besonders nachteilig. Die Auswertung der Daten der großen amerikanischen Sleep Heart Health Study ergab z.B. eine um den Faktor zwei erhöhte Wahrscheinlichkeit für das Vorliegen einer Herzinsuffizienz [58]. In mehreren prospektiven randomisierten Studien verbesserte die CPAP-Therapie die linksventrikuläre Ejektionsfraktion (LVEF) bei Patienten mit Herzinsuffizienz und gleichzeitig vorliegender OSA [59]. Diese Befunde sind allgemein akzeptiert, auch wenn eine gut kontrollierte schottische Studie dies nicht replizieren konnte [60]. In einer retrospektiven Untersuchung an über 30000 Patienten mit neu diagnostizierter Herzinsuffizienz war die CPAP-Therapie nach Adjustierung für andere Risikofaktoren mit einem deutlich besseren Überleben verbunden ( $\bullet$ Abb.2). Bei nur einem geringen Anteil der Patienten wurde überhaupt eine Untersuchung hinsichtlich OSA durchgeführt [61].

Die Leitlinien der European Society of Cardiology stellen in der Therapie der Herzinsuffizienz die Gewichtreduktion, Tabakentwöhnung und Alkoholverzicht mit jeweils starker Empfehlung (Class of recommendation 1) voran [62]. Bei dokumentierter OSA wird eine CPAP-Therapie empfohlen (Class of recommendation 2a). Laufende randomisierte Studien werden hier wichtige Informationen liefern.

Diastolische Dysfunktion Wir konnten kürzlich an einer größeren Kohorte von Patienten mit kardiovaskulären Risikofaktoren zeigen, dass eine bisher nicht diagnostizierte OSA ein unabhängiger Risikofaktor für eine diastolische Dysfunktion darstellt [63]. Auch in anderen Kollektiven war dieser Zusammenhang unabhängig vom Vorliegen eines erhöhten Blutdruckes. In einer gut kontrollierten randomisierten, plazebo-kontrollierten doppelt blinden Studie bei normotensiven OSA-Patienten ohne KV-Erkrankungen verbesserte die CPAP-Therapie die diastolische Funktion [64]. Verschiedene weitere Studien kommen zu ähnlichen Ergebnissen.
Tab. 2 Einige positive Effekte der CPAP-Therapie auf Mechanismen der kardiovaskulären Komorbidität.

\begin{tabular}{l} 
Besserung der Endothelfunktion \\
Steigerung der NO-Produktion \\
Steigerung des postischämischen Blutflusses \\
\hline Abnahme von Endothelin-1 \\
\hline Abnahme von Entzündungsmediatoren (CRP, TNF...) \\
\hline Abnahme freier Sauerstoffradikale \\
\hline Abnahme von Fibrinogen, Hyperkoagulation \\
\hline Abnahme der Thrombozytenaktivität und Aggregation \\
\hline Zunahme endothelialer Vorläuferzellen \\
Verbesserung des Glukosestoffwechsels \\
Abnahme der sympathischen Aktivität \\
Abnahme der Gefäßsteifigkeit \\
Abnahme der Intimadicke \\
verbesserte Funktion von „clock genes“ (Uhren-Genen)
\end{tabular}

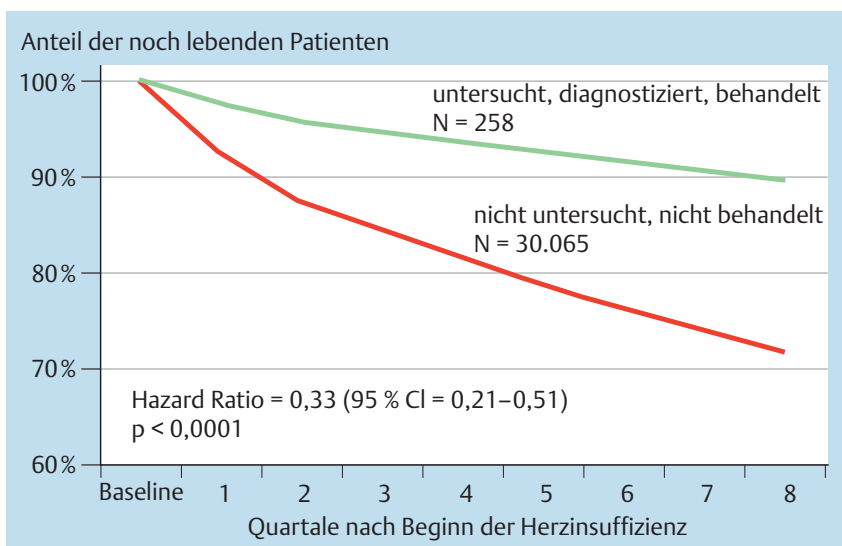

Abb.2 Diagnostik und Therapie nächtlicher Atemstörungen bessert das Überleben von Patienten mit Herzinsuffizienz (modifiziert nach [61]).

Ischämische Herzerkrankung Obwohl mehrere Querschnittsstudien eine Assoziation zwischen OSA und ischämischer Herzerkrankung zeigten, ist der Wert der Studien überwiegend durch Selektionsbias, kleine Patientenzahlen und fehlende Kontrollgruppen eingeschränkt [6]. Die oben erwähnte sehr große Sleep Heart Health Study wies ein moderates, aber signifikant erhöhtes Risiko von 1,2 (95\%, CI 0,99-1,62) für berichtete Herzerkrankungen bei Patienten mit einem AHI > 30/h im Vergleich zu Patienten mit einem $\mathrm{AHI}<1,5 / \mathrm{h}$ auf [65].

Diese Assoziation wurde durch eine weitere Studie gestützt, die 308 Patienten mit arterieller Hypertonie oder einer ischämischen Herzkrankheit über 7 Jahre nachbeobachtete [66]. Die Patienten mit OSA hatten ein 4,9-fach erhöhtes Risiko für eine ischämische Herzerkrankung unabhängig von Alter, Geschlecht, Blutdruck, Diabetes und Raucherstatus. In einer retrospektiven Studie von Patienten mit OSA, bei denen eine perkutane koronare Intervention (PCI) durchgeführt wurde, war die kardiale Mortalität in der CPAP-Gruppe im Vergleich zu der nicht behandelten Gruppe erhöht [67]. Die wichtige Frage, ob wir im Alltag systematisch asymptomatische OSA-Patienten mit ischämischer Herzerkrankung mittels CPAP therapieren sollen, kann erst durch laufende randomisierte, kontrollierte Studien beantwortet werden $[6,68]$. Schlaganfall In der oben erwähnten Studie (SHHS) war bei einem AHI>19/h die adjustierte Ereignis-Rate für einen Schlaganfall mit 2,9 gegenüber Patienten mit einem niedrigen Apnoe-/ Hypopnoe-Index in der fast 9-jährigen Nachbeobachtung erhöht [69]. 
In einer der ersten Studien bei Patienten mit Schlaganfall und OSA konnte eine CPAP-Therapie im Reha-Setting effektiv durchgeführt werden [70]. In einer aktuellen Studie bei Patienten mit Schlaganfall und mäßiger bis schwerer OSA war nach einer Nachbeobachtung von 7 Jahren bei Patienten, die eine CPAP-Therapie erhalten hatten, das Risiko, KV-Ereignisse zu erleiden, um etwa $70 \%$ reduziert [71]. Die Therapieentscheidung wird im Alltag auch dadurch erschwert, dass ein Schlaganfall akut eine OSA begünstigt, die sich dann im weiteren Verlauf wieder deutlich bessern kann [72].

Große Gefäße Aufgrund des eindeutigen Zusammenhanges zwischen OSA und arteriellem Hypertonus auf der einen Seite und arteriellem Hypertonus und Aortendissektion auf der anderen Seite ist es nicht verwunderlich, dass die OSA eng mit dem Auftreten von Aortenaneurysmen und der Aortendissektion verbunden ist [73]. Auch die Intimadicke der Arteria carotis und die arterielle Pulswellengeschwindigkeit ist bei OSA pathologisch und wird durch CPAP positiv beeinflusst $[6,73]$.

Vorhofflimmern Wesentliche Risikofaktoren für das Auftreten von Vorhofflimmern sind höheres Alter, arterieller Hypertonus und Herzinsuffizienz. Daher ist es plausibel, dass Vorhofflimmern bei OSA häufiger auftritt [7]. Auch der plötzliche Herztod tritt bei OSA häufiger in der Nacht auf [74]. Interventionsstudien zeigen eine Reduktion von Vorhofflimmern unter CPAP, sind allerdings bisher nicht randomisiert [75].

Thrombembolische Erkrankungen Die oben geschilderten Veränderungen der Endothelfunktion und der Gerinnung haben das Potenzial, auch thrombembolische Erkrankungen (Lungenembolie und/oder Beinvenenthrombose) auszulösen. Tatsächlich war in einer Fall-Kontroll-Studie die Prävalenz der OSA bei Patienten mit thrombembolischer Erkrankung erhöht [76]. Dies war auch noch nach Berücksichtigung von verschiedenen Risikofaktoren (Confoundern) bei den Frauen, nicht jedoch bei den Männern der Fall.

Pulmonale Hypertonie Schlafbezogene Atemstörungen sind als Ursache der PH bei Lungenerkrankungen in den aktuellen Leitlinien berücksichtigt [77]. Die Pulmonale Hypertonie infolge einer OSA ist meist mild [78]. Eine klinisch relevante pulmonale Hypertonie ist jedoch häufiger zu finden, wenn gleichzeitig eine COPD, ein Adipositas-Hypoventilations-Syndrom oder auch eine diastolische Dysfunktion vorliegt [6]. In kleineren Untersuchungen reduziert die CPAP-Therapie den pulmonalarteriellen Druck insbesondere bei Patienten mit ausgeprägter OSA [78]. Bei Patienten mit schwerer idiopathischer pulmonal arterieller Hypertonie (IPAH) kann ähnlich wie bei der Herzinsuffizienz eine CSA infolge der kardialen Situation auftreten [78].

\section{Therapeutische Ansätze neben CPAP}

\section{$\nabla$}

Auch wenn der Zusammenhang zwischen OSA und arteriellem Hypertonus eindeutig gesichert ist und ein Zusammenhang zwischen OSA und KV-Erkrankungen sehr gut belegt ist, gibt es eine Reihe von etablierten KV-Risikofaktoren, die der Therapie gut zugänglich sind. Daher werden hier drei Therapieansätze besprochen, die nicht nur das KV-Risiko deutlich senken, sondern auch einen positiven Effekt auf die OSA haben.

\section{Gewichtsreduktion}

Während wir über die Effekte der OSA auf das metabolische Syndrom diskutieren, sollten wir uns klarmachen, dass es mindestens 3 große, gut kontrollierte randomisierte Studien gibt, die eine klinisch bedeutende Körpergewichtsreduktion von etwa 20 $\mathrm{kg}$ durch intensive Beratung und niedrigenergetische Diät zeigen. Die Körpergewichtsreduktion war von einer eindeutigen Verbesserung der OSA bei $60 \%$ der Patienten begleitet [79]. Weiterhin werden alle Aspekte des metabolischen Syndroms eindeutig durch Gewichtsreduktion verbessert, die auch durch bariatrische Chirurgie zu erreichen ist. Studien zur Langzeitbehandlung werden durchgeführt, um den Gewichtsverlust als primäre Behandlungsstrategie der obstruktiven Schlafapnoe zu prüfen.

Bei gut belegten positiven Effekten der Gewichtsreduktion auf die KV-Morbidität und -Mortalität sowie die meist ausreichende Besserung der OSA unter Gewichtsreduktion, müssen wir uns fragen, wieso wir diese Therapie in Deutschland kaum anwenden.

\section{Tabakentwöhnung}

Tabakrauchen ist nicht nur ein wesentlicher, gut zu therapierender Risikofaktor für kardiovaskuläre Erkrankungen sondern begünstigt auch das Auftreten der OSA [80,81]. Ebenso zeigte eine Longitudinalstudie, dass der Beginn des Rauchens einen unabhängigen Risikofaktor für die Entwicklung primären Schnarchens mit einer odds ratio von 2,2 darstellt [82]. Die zugrundeliegende Pathophysiologie umfasst pharyngeale Entzündung und Reflexänderungen der oberen Atemwege.

\section{Körperliches Training}

Seit langem ist ein Zusammenhang zwischen Bewegungsarmut, Adipositas und OSA bekannt. In gut kontrollierten Querschnittsstudien war Bewegungsarmut ein von Übergewicht unabhängiger Risikofaktor für die Schwere der OSA [83]. Dieser Zusammenhang wird wahrscheinlich über eine Erhöhung des pharyngalen Muskeltonus durch Bewegung vermittelt.

\section{Interessenkonflikt}

$\nabla$

S. Andreas: Honorare für Vorträge von Heinen \& Löwenstein, Teilnahme an einer Studie von ResMed.

M. Meier: Teilnahme an einer Studie von ResMed.

\section{Literatur}

1 Bradley TD, Brown IG, Grossman RF et al. Pharyngeal size in snorers, nonsnorers, and patients with obstructive sleep apnea. N Engl J Med 1986; 315: $1327-1331$

2 Peppard PE, Young T, Palta $M$ et al. Longitudinal study of moderate weight change and sleep-disordered breathing. JAMA 2000; 284: 3015-3021

3 Young T, Palta M, Dempsey J et al. The occurrence of sleep-disordered breathing among middle-aged adults. N Engl J Med 1993; 328: $1230-1235$

4 Duran J, Esnaola S, Rubio R et al. Obstructive sleep apnea-hypopnea and related clinical features in a population-based sample of subjects aged 30 to 70 yr. Am J Respir Crit Care Med 2001; 163: 685-689

5 Owan TE, Hodge DO, Herges RM et al. Trends in prevalence and outcome of heart failure with preserved ejection fraction. N Engl J Med 2006; 355: $251-259$

6 Lüthje L, Andreas S. Obstructive sleep apnea and coronary artery disease. Sleep Medicine Review 2008; 12: 19-31

7 Bradley TD, Floras JS. Obstructive sleep apnoea and its cardiovascular consequences. Lancet 2009; 373: 82 -93

8 Hung J, Whitford EG, Parsons RW et al. Association of sleep apnoea with myocardial infarction in men. Lancet 1990; 336: 261 -264

9 Buchner S, Greimel T, Hetzenecker A et al. Natural course of sleep-disordered breathing after acute myocardial infarction. Eur Respir J 2012: epub ahead of print

10 Schulz R, Blau A, Borgel J et al. Sleep apnoea in heart failure - results of a German survey. Eur Respir J 2007; 29: 1201 - 1205 
11 Bradley TD, Floras JS. Sleep apnea and heart failure: Part II: central sleep apnea. Circulation 2003; 107: 1822-1826

12 Morgan BJ, Denahan T, Ebert TJ. Neurocirculatory consequences of negative intrathoracic pressure vs. asphyxia during voluntary apnea. J Appl Physiol 1993; 74: 2969-2975

13 Andreas S, Bingeli C, Mohacsi P et al. Nasal oxygen and muscle sympathetic nerve activity in heart failure. Chest 2003; 123: 366 - 371

14 Davies RJ, Belt PJ, Roberts SJ et al. Arterial blood pressure responses to graded transient arousal from sleep in normal humans. J Appl Physiol 1993; 74: $1123-1130$

15 Andreas S, Werner GS, Sold G et al. Doppler echocardiographic analysis of cardiac flow during the Mueller manoeuver. Eur J Clin Invest 1991; 21: $72-76$

16 Scharf SM. Cardiovascular effects of airways obstruction. Lung 1991; 169: $1-23$

17 Andreas $S$, Richter DW. Changes in heart rate during obstructive sleep apnea. Eur Respir J 1993; 6: 1074

18 Bonsignore MR, Romano S, Marrone $O$ et al. Different heart rate patterns in obstructive apneas during NREM sleep. Sleep 1997; 20: $1167-1174$

19 Andreas S, Hajak G, Natt P et al. ST segmental changes and arrhythmias in obstructive sleep apnea. Pneumol 1991; 45: 720-724

20 Andreas $S$, von Breska B, Schaumann A et al. Obstructive sleep apnoea and signal averaged electrocardiogram. Eur Respir J 1995; 8: 546-550

21 Franklin KA, Nilsson JB, Sahlin C et al. Sleep apnoea and nocturnal angina. Lancet 1995; 345: $1085-1087$

22 Valipour A, McGown AD, Makker H et al. Some factors affecting cerebral tissue saturation during obstructive sleep apnoea. Eur Respir J 2002; 20: $444-450$

23 Podszus T, Bauer W, Mayer J et al. Sleep apnea and pulmonary hypertension. Klin Wochenschr 1986; 64: 131-134

24 Dyugovskaya L, Lavie P, Lavie L. Increased adhesion molecules expression and production of reactive oxygen species in leukocytes of sleep apnea patients. Am J Respir Crit Care Med 2002; 165: 934-939

25 Schulz R, Mahmoudi S, Hattar K et al. Enhanced release of superoxide from polymorphonuclear neutrophils in obstructive sleep apnea. Impact of continuous positive airway pressure therapy. Am J Respir Crit Care Med 2000; 162: 566-570

26 Ryan S, Taylor CT, McNicholas WT. Predictors of elevated nuclear factorkappaB-dependent genes in obstructive sleep apnea syndrome. Am J Respir Crit Care Med 2006; 174: 824-830

27 Minoguchi K, Tazaki T, Yokoe T et al. Elevated production of tumor necrosis factor-alpha by monocytes in patients with obstructive sleep apnea syndrome. Chest 2004; 126: $1473-1479$

28 Minoguchi K, Yokoe T, Tazaki T et al. Increased carotid intima-media thickness and serum inflammatory markers in obstructive sleep apnea. Am J Respir Crit Care Med 2005; 172: 625-630

29 Allahdadi KJ, Walker BR, Kanagy NL. Augmented endothelin vasoconstriction in intermittent hypoxia-induced hypertension. Hypertension 2005; 45: 705-709

30 Phillips BG, Narkiewicz K, Pesek CA et al. Effects of obstructive sleep apnea on endothelin-1 and blood pressure. J Hypertens 1999; 17: 61 -66

31 Grimpen F, Kanne P, Schulz E et al. Endothelin-1 plasma levels are not elevated in patients with obstructive sleep apnea. Eur Respir J 2000; 15: $320-325$

32 Wessendorf TE, Thilmann AF, Wang YM et al. Fibrinogen levels and obstructive sleep apnea in ischemic stroke. Am J Respir Crit Care Med 2000; 162: 2039-2042

33 Mehra R, Xu F, Babineau DC et al. Sleep-disordered breathing and prothrombotic biomarkers: cross-sectional results of the Cleveland Family Study. Am J Respir Crit Care Med 2010; 182: 826-833

34 Phillips CL, McEwen BJ, Morel-Kopp MC et al. Effects of continuous positive airway pressure on coagulability in obstructive sleep apnoea: a randomised, placebo-controlled crossover study. Thorax 2012; 67: $639-644$

35 Carlson JT, Hedner J, Elam $M$ et al. Augmented resting sympathetic activity in awake patients with obstructive sleep apnea. Chest 1993; 103: $1763-1768$

36 Somers VK, Dyken ME, Clary MP et al. Sympathetic neural mechanisms in obstructive sleep apnea. J Clin Invest 1995; 96: 1897-1904

37 Donadio V, Liguori $R$, Vetrugno $R$ et al. Daytime sympathetic hyperactivity in OSAS is related to excessive daytime sleepiness. J Sleep Res 2007; 16: $327-332$

38 Andreas $S$, Eichele G. Sleep apnea - time to consider clock genes. Eur Respir J 2008; 32: 1-2
39 Andreas S, Anker SD, Scanlon PD et al. Neurohumoral activation as a link to systemic manifestation of chronic lung disease. Chest 2005; 128: $3618-3624$

40 Ip MS, Lam B, Chan LY et al. Circulating nitric oxide is suppressed in obstructive sleep apnea and is reversed by nasal continuous positive airway pressure. Am J Respir Crit Care Med 2000; 162: 2166-2171

41 Takase B, Akima T, Uehata $A$ et al. Effect of chronic stress and sleep deprivation on both flow-mediated dilation in the brachial artery and the intracellular magnesium level in humans. Clin Cardiol 2004; 27: 223 227

42 Patt BT, Jarjoura D, Haddad DN et al. Endothelial dysfunction in the microcirculation of patients with obstructive sleep apnea. Am J Respir Crit Care Med 2010; 182: 1540-1545

43 Drager LF, Bortolotto LA, Figueiredo AC et al. Effects of continuous positive airway pressure on early signs of atherosclerosis in obstructive sleep apnea. Am J Respir Crit Care Med 2007; 176: 706 - 712

44 Peters $A$, Kubera B, Hubold $C$ et al. The selfish brain: stress and eating behavior. Front Neurosci 2011; 5: 74

45 Schulz R, Eisele HJ, Reichenberger F et al. [Obstructive sleep apnoea and metabolic syndrome]. Pneumologie 2008; 62: 88-91

46 Sharma SK, Agrawal S, Damodaran D et al. CPAP for the metabolic syndrome in patients with obstructive sleep apnea. N Engl J Med 2011; 365: $2277-2286$

47 Andreas S. Nocturnal insights in chronic heart failure. Eur Heart J 1999; 20: $1140-1141$

48 Spicuzza L, Bernardi L, Calciati A et al. Autonomic modulation of heart rate during obstructive versus central apneas in patients with sleepdisordered breathing. Am J Respir Crit Care Med 2003; 167: 902 - 910

49 Brooks D, Horner RL, Kozar LF et al. Obstructive sleep apnea as a cause of systemic hypertension. Evidence from a canine model [see comments]. J Clin Invest 1997; 99: 106-109

50 Bazzano LA, Khan Z, Reynolds K et al. Effect of nocturnal nasal continuous positive airway pressure on blood pressure in obstructive sleep apnea. Hypertension 2007; 50: 417-423

51 Haentjens P, Van Meerhaeghe A, Moscariello A et al. The impact of continuous positive airway pressure on blood pressure in patients with obstructive sleep apnea syndrome: evidence from a meta-analysis of placebo-controlled randomized trials. Arch Intern Med 2007; 167: $757-764$

52 Montserrat JM, Garcia-Rio F, Barbe F. Diagnostic and therapeutic approach to nonsleepy apnea. Am J Respir Crit Care Med 2007; 176: 6-9

53 Marin JM, Carrizo SJ, Vicente E et al. Long-term cardiovascular outcomes in men with obstructive sleep apnoea-hypopnoea with or without treatment with continuous positive airway pressure: an observational study. Lancet 2005; 365: 1046-1053

54 Young T, Finn L, Peppard PE et al. Sleep disordered breathing and mortality: eighteen-year follow-up of the Wisconsin sleep cohort. Sleep 2008; 31: $1071-1078$

55 Buchner NJ, Sanner BM, Borgel J et al. Continuous positive airway pressure treatment of mild to moderate obstructive sleep apnea reduces cardiovascular risk. Am J Respir Crit Care Med 2007; 176: 1274-1280

56 Lavie $P$, Lavie $L$. Unexpected survival advantage in elderly people with moderate sleep apnoea. J Sleep Res 2009; 18: 397-403

57 Punjabi NM, Caffo BS, Goodwin JL et al. Sleep-disordered breathing and mortality: a prospective cohort study. PLoS Med 2009; 6: e1000132

58 Shahar E, Whitney CW, Redline S et al. Sleep-disordered breathing and cardiovascular disease: cross-sectional results of the Sleep Heart Health Study. Am J Respir Crit Care Med 2001; 163: 19-25

59 Kaneko Y, Floras JS, Usui $\mathrm{K}$ et al. Cardiovascular effects of continuous positive airway pressure in patients with heart failure and obstructive sleep apnea. N Engl J Med 2003; 348: 1233 - 1241

60 Smith LA, Vennelle M, Gardner RS et al. Auto-titrating continuous positive airway pressure therapy in patients with chronic heart failure and obstructive sleep apnoea: a randomized placebo-controlled trial. Eur Heart J 2007; 28: 1221 - 1227

61 Javaheri S, Caref EB, Chen E et al. Sleep apnea testing and outcomes in a large cohort of Medicare beneficiaries with newly diagnosed heart failure. Am J Respir Crit Care Med 2011; 183: 539-546

62 Dickstein K, Cohen-Solal A, Filippatos G et al. ESC Guidelines for the diagnosis and treatment of acute and chronic heart failure 2008: the Task Force for the Diagnosis and Treatment of Acute and Chronic Heart Failure 2008 of the European Society of Cardiology. Developed in collaboration with the Heart Failure Association of the ESC (HFA) and endorsed by the European Society of Intensive Care Medicine (ESICM). Eur Heart J 2008; 29: 2388-2442 
63 Wachter R, Luthje L, Klemmstein D et al. Impact of obstructive sleep apnoea on diastolic function. Eur Respir J 2012: in press

64 Arias MA, Garcia-Rio F, Alonso-Fernandez A et al. Obstructive sleep apnea syndrome affects left ventricular diastolic function: effects of nasal continuous positive airway pressure in men. Circulation 2005; 112: $375-383$

65 Nieto FJ, Young TB, Lind BK et al. Association of sleep-disordered breathing, sleep apnea, and hypertension in a large community-based study. Sleep Heart Health Study. JAMA 2000; 283: 1829-1836

66 Peker Y, Carlson J, Hedner J. Increased incidence of coronary artery disease in sleep apnoea: a long-term follow-up. Eur Respir J 2006; 28: 596-602

67 Cassar A, Morgenthaler TI, Lennon RJ et al. Treatment of obstructive sleep apnea is associated with decreased cardiac death after percutaneous coronary intervention. J Am Coll Cardiol 2007; 50: 1310-1314

68 Peker Y, Glantz H, Thunstrom E et al. Rationale and design of the Randomized Intervention with CPAP in Coronary Artery Disease and Sleep Apnoea-RICCADSA trial. Scand Cardiovasc J 2009; 43: 24-31

69 Redline S, Yenokyan G, Gottlieb DJ et al. Obstructive sleep apnea-hypopnea and incident stroke: the sleep heart health study. Am J Respir Crit Care Med 2010; 182: 269-277

70 Wessendorf TE, Wang YM, Thilmann AF et al. Treatment of obstructive sleep apnoea with nasal continuous positive airway pressure in stroke. Eur Respir J 2001; 18: 623-629

71 Martinez-Garcia MA, Campos-Rodriguez F, Soler-Cataluna JJ et al. Increased incidence of nonfatal cardiovascular events in stroke patients with sleep apnoea: effect of CPAP treatment. Eur Respir J 2012; 39: 906-912

72 Dyken ME, Im KB. Obstructive sleep apnea and stroke. Chest 2009; 136: $1668-1677$

73 Mason RH, Ruegg G, Perkins J et al. Obstructive sleep apnea in patients with abdominal aortic aneurysms: highly prevalent and associated with aneurysm expansion. Am J Respir Crit Care Med 2011; 183 : 668-674

74 Gami AS, Howard DE, Olson EJ et al. Day-night pattern of sudden death in obstructive sleep apnea. N Engl J Med 2005; 352: 1206 - 1214

75 Kanagala R, Murali NS, Friedman PA et al. Obstructive sleep apnea and the recurrence of atrial fibrillation. Circulation 2003; 107: 2589-2594

76 Arzt M, Luigart R, Schum C et al. Sleep-disordered breathing in deep vein thrombosis and acute pulmonary embolism. Eur Respir J 2012; 40: $919-924$

77 Hoeper MM, Andreas S, Bastian A et al. Pulmonary hypertension due to chronic lung disease: Updated Recommendations of the Cologne Consensus Conference 2011. Int J Cardiol 2011; 154: S45 - S53

78 Schulz R, Eisele HJ, Weissmann $N$ et al. Pulmonale Hypertonie und schlafbezogene Atemstörung. Pneumologie 2005; 59: 270-274

79 Johansson K, Neovius $M$, Lagerros YT et al. Effect of a very low energy diet on moderate and severe obstructive sleep apnoea in obese men: a randomised controlled trial. Bmj 2009; 339: b4609

80 Young T, Skatrud J, Peppard PE. Risk factors for obstructive sleep apnea in adults. JAMA 2004; 291: 2013-2016

81 Franklin KA, Gislason T, Omenaas E et al. The influence of active and passive smoking on habitual snoring. Am J Respir Crit Care Med 2004; 170: 799-803

82 Knuiman M, James A, Divitini M et al. Longitudinal study of risk factors for habitual snoring in a general adult population: the Busselton Health Study. Chest 2006; 130: 1779-1783

83 Peppard PE, Young T. Exercise and sleep-disordered breathing: an association independent of body habitus. Sleep 2004; 27: 480-484

84 Shamsuzzaman AS, Gersh BJ, Somers VK. Obstructive sleep apnea: implications for cardiac and vascular disease. JAMA 2003; 290: 19061914 